\title{
Regularity of the Interband Light Absorption Coefficient
}

\author{
M Krishna \\ Institute of Mathematical Sciences \\ Taramani Chennai 600113 \\ India
}

24 November 2008

\begin{abstract}
In this paper we consider the Interband Light Absorption Coefficient (ILAC), in a symmetric form, in the case of random operators on the $d$-dimensional lattice. We show that the symmetrized version of ILAC is either continuous or has a component which has the same modulus of continuity as the density of states.
\end{abstract}

\section{Introduction}

In the theory of disordered systems, one of the quantities that is widely studied is the integrated density of states, whose continuity properties and its behaviour near band edges (Lifshitz tails) were of great interest. Another quantity that is of interest is the interband light absorption coefficient ILAC, which is an important quantitative characteristic of semiconductors.

The literature on the density of states is vast so we refer the reader to the books [3], [4], [17] and the review [8]. The continuity properties of the density of states and its Lifshitz tails behaviour in various models is widely understood. The physics literature is abound with works on the ILAC starting from [7] and for example [1]. On the other hand rigorous work in this area seem to be minimal, see for example [10], [11], [13], [12]. 
We consider a borel probability space $(\Omega, \mathcal{B}, \mathbb{P})$ with $\mathbb{Z}^{d}$ acting on $\Omega$ such that $\mathbb{P}$ is invariant and ergodic with respect to this action. Let $V: \Omega \rightarrow \mathbb{R}^{\mathbb{Z}^{d}}$ (so that each $V(n)$ is measurable). We consider a self-adjoint operator $H_{0}=$ $\Delta$ (given in equation (3) ) on $\ell^{2}\left(\mathbb{Z}^{d}\right)$ and consider the family of operators,

$$
H_{\omega}^{ \pm}=H_{0} \pm V^{\omega},\left(V^{\omega} u\right)(n)=V^{\omega}(n) u(n), u \in \ell^{2}\left(\mathbb{Z}^{d}\right) .
$$

We denote by $\delta_{n}$ the elements of the standard basis of $\ell^{2}\left(\mathbb{Z}^{d}\right)$ in the rest of the paper.

We define the density of states measures $n_{ \pm}$associated with $H_{\omega}^{ \pm}$by

$$
n_{ \pm}=\mathbb{E}\left(\left\langle\delta_{0}, E_{H_{\omega}^{ \pm}}(\cdot) \delta_{0}\right\rangle\right) .
$$

We denote by $U_{i}, i \in \mathbb{Z}^{d}$ the unitary operators $\left(U_{i} u\right)(n)=u(n-i), u \in$ $\ell^{2}\left(\mathbb{Z}^{d}\right)$.

Hypothesis 1.1. 1. The random potential $V^{\omega}$ satisfies $U_{i}^{*} V^{\omega} U_{i}=V^{T_{i} \omega}$, where $T_{i}$ is the action of $e_{i}$ on $\Omega$.

2. There is a bijection $R$ of $\Omega$ to itself such that $V^{R \omega}=-V^{\omega}$ and $\mathbb{P}$ is invariant under $R$.

3. The operators $H_{\omega}^{ \pm}$are self-adjoint with a common dense domain for a set of full measure in $\omega$.

4. The density of states measures $n_{ \pm}$are continuous.

Examples 1.2. Here are two extreme examples of operators satisfying the above conditions. Of course there are many more of various varaities.

1. The Anderson Model:

$$
V^{\omega}(n)=\omega(n),(\Delta u)(n)=\sum_{|i|=1} u(n+i), \quad u \in \ell^{2}\left(\mathbb{Z}^{d}\right)
$$

and $R \omega=-\omega$ and $\mathbb{P}=\times \mu$ with a probability measure $\mu$ on $\mathbb{R}$. If $\mu$ is continuous, then the density of states is continuous. We take $\mu$ to satisfy $\mu(B)=\mu(-B)$ for all borel subsets of $\mathbb{R}$ and take $R \omega=-\omega$. Then $\mathbb{P}$ is invariant under $R$.

2. The Almost Mathieu model: Take $d=1$ and take $\Omega=\mathbb{T}, V^{\omega}(n)=$ $\lambda \cos (\alpha n+\omega), R \omega=\omega+\pi$ and $\mathbb{P}$ the rotation invariant measure on $\mathbb{T}$. The density of states of this model is absolutely continuous, when $\alpha$ is not rational and for $|\lambda| \neq 2$, see [2]. 
Remark 1.3. We note that, using the definition of $H_{\omega}^{ \pm}$and $V^{\omega}$ and the bijection $R$ mentioned in the Hypothesis 1.1, that

$$
H_{\omega}^{-}=H_{R \omega}^{+}, \quad H_{\omega}^{+}=H_{R \omega}^{-} .
$$

Therefore if $\mathbb{P}$ satisfies Hypothesis 1.1 (2), then for any integrable function $f$ of $\omega$, we have

$$
\mathbb{E}(f(\omega))=\mathbb{E}(f(R \omega)) .
$$

The immediate consequence of our hypothesis is the equality of spectra of $H_{\omega}^{ \pm}$.

Theorem 1.4. Let $H_{\omega}^{ \pm}$be as in Hypothesis 1.1. Then we have

$$
\sigma\left(H_{\omega}^{+}\right)=\sigma\left(H_{\omega}^{-}\right) \text {, a.e. } \omega .
$$

Proof: Under the assumptions of Hypothesis 1.1, it is well known that the spectrum of the associated operators $\sigma\left(H_{\omega}^{ \pm}\right)$are constant sets almost everywhere (Proposition V.2.4, Carmona-Lacroix [3]). The Hypothesis 1.1(2) implies that $H_{\omega}^{+}=H_{R \omega}^{-}$and also that the support of $\mathbb{P}$ is invariant under $R$. Therefore we have

$$
\sigma\left(H_{\omega}^{+}\right)=\sigma\left(H_{R \omega}^{+}\right)=\sigma\left(H_{\omega}^{-}\right), \text {a.e. } \omega
$$

proving the result.

We consider $H_{\omega}^{ \pm}$as in equation (1), their spectral measures $E_{H_{\omega}^{ \pm}}$and define the measure $\rho$ as follows.

$$
\rho=\mathbb{E}\left(\left\langle\delta_{0}, E_{H_{\omega}^{+}}(\cdot) E_{H_{\omega}^{-}}(\cdot) \delta_{0}\right\rangle\right)
$$

on $\mathbb{R}^{2}$. (Let

$$
\mathcal{I}=\mathbb{R} \cup\{(a, b]: a, b \in \mathbb{R}\} \cup\{(a, \infty): a \in \mathbb{R}\} \cup\{(-\infty, a]: a \in \mathbb{R}\} .
$$

This collection of sets forms a boolean semi-algebra on $\mathbb{R}$. We then consider the boolean semialgebra $\mathcal{I} \times \mathcal{I}$ and there define the set function $\rho$ by

$$
\rho\left(\cup_{i=1}^{k} I_{i} \times J_{i}\right)=\sum_{i=1}^{k} \mathbb{E}\left(\left\langle\delta_{0}, E_{H_{\omega}^{+}}\left(I_{i}\right) E_{H_{\omega}^{-}}\left(J_{i}\right) \delta_{0}\right\rangle\right), I_{i}, J_{i} \in \mathcal{I},
$$

where the $\left\{I_{i} \times J_{i} i=1, \ldots, k\right\}$ are mutually disjoint rectangles. Then this $\rho$, takes values in $[0,1]$ and satisfies $\rho(\mathbb{R} \times \mathbb{R})=1$. The positivity of $\rho$ follows 
from Proposition 2.5 (2), below and since intersection of rectangles of the form considered are again rectangles of the same form, $\rho$ is also seen to be well defined. Hence it extends to a unique probability measure on the boolean algebra generated by $\mathcal{I} \times \mathcal{I}$, see Exercises 1.4.4 - 1.4.6 and Proposition 1.4.7 of [15]. The unique extension of this to a probability measure on the borel $\sigma$-algebra on $\mathbb{R}^{2}$ is again standard, see Proposition 2.5.1 [15].)

Suppose $H_{\omega, \Lambda}^{ \pm}$are the restrictions of $H_{\omega}^{ \pm}$to $\Lambda \subset \mathbb{Z}^{d}$ and $\lambda_{ \pm}, u_{\lambda_{ \pm}}$are eigenvalues and eigen functions of $H_{\Lambda, \omega}^{ \pm}$.

It would be nice to show (as done in Kirsch-Pastur [10] for some models on $L^{2}\left(\mathbb{R}^{d}\right)$ ), that $\rho$ is the a.e. (weak) limit of the measures

$$
\rho_{\Lambda, \omega}=\frac{1}{|\Lambda|} \sum_{\lambda_{ \pm} \in \sigma\left(H_{\omega, \Lambda}^{ \pm}\right)}\left|\left\langle u_{\lambda_{+}}, v_{\lambda_{-}}\right\rangle\right|^{2} \delta_{\lambda_{+}, \lambda_{-}},
$$

as $\Lambda \rightarrow \mathbb{Z}^{d}$. Then, the ILAC could be taken to be the limit of the distribution functions

$$
A_{a s, \Lambda}(\lambda)=\sum_{\lambda_{+}+\lambda_{-} \leq \lambda} \rho_{\Lambda, \omega}\left(\lambda_{+}, \lambda_{-}\right),
$$

as done in the continuum models.

We do not really need this point wise statement here, but only some weaker version, which we prove in the next section.

Let $T$ be a transformation from $\mathbb{R}^{2}$ to itself given by the matrix

$$
\frac{1}{\sqrt{2}}\left(\begin{array}{cc}
1 & 1 \\
1 & -1
\end{array}\right)
$$

Then $T$ is an orthogonal matrix with $T^{-1}=T$ and we have

$$
T\left(\begin{array}{l}
\lambda_{1} \\
\lambda_{2}
\end{array}\right)=\left(\begin{array}{c}
\frac{\lambda_{1}+\lambda_{2}}{\sqrt{2}} \\
\frac{\lambda_{1}-\lambda_{2}}{\sqrt{2}}
\end{array}\right) .
$$

Using these we define:

Definition 1.5. We consider the measure $\rho$ defined in equation (5) and set the asymmetric ILAC as:

$$
A_{a s}(\lambda)=\nu((-\infty, \lambda]), \text { where } \nu(B)=\rho \circ T^{-1}(B \times \mathbb{R})
$$

and the symmetric ILAC as :

$A_{s}(\lambda)=\sigma((-\infty, \lambda])$, where $\sigma(B)=\frac{1}{2}\left(\rho \circ T^{-1}(B \times \mathbb{R})+\rho \circ T^{-1}(\mathbb{R} \times B)\right)$. 
In the above definitions and in our model we have dropped all the physical constants and also have dropped the band gap $E_{g}$ that customarily appears in the definition since they play no role in the regularity properties as seen in the proofs of our theorems.

The reason we consider a symmetrized version of ILAC is that, in the case of disordered models where the spectrum is symmetric about 0 , if $\lambda$ is in the spectrum then $-\lambda$ is also in the spectrum. Therefore given a $E$ we can have $\lambda^{+}+\lambda^{-}=E$ and also $\lambda_{+}-\left(\tilde{\lambda}_{-}\right)=E$ (of course $\tilde{\lambda}_{-}$would be $-\lambda_{-}$). Therefore in the definition of the finite distribution functions in equation (7) we could also have taken the sum over $\lambda_{+}-\lambda_{-} \leq E$. The distribution functions, however differ for these two different definitions. Therefore it might be more meaningful to take a symmetric definition.

\section{Regularity Properties}

In this section we show a regularity of a symmetrized ILAC. The idea behind the proofs is the following. The ILAC is the distribution function of a marginal of a twisted two dimensional measure, whose marginals are the density of states. This measure $\rho$ itself acquires the regularity of the density of states, however it is possible that a marginal of the twisted version might have an atomic component. This is possible only if the twisted measure itself has its support (not the topological support) $\Sigma_{1} \cup \Sigma_{2}$, with $\Sigma_{1}$ being a subset of a straight line which is disjoint from $\Sigma_{2}$. If this happens then on the straight line containing $\Sigma_{1}$, the twisted measure must be as regular as the density of states. This is precisely our conclusion.

We note here that in the proofs below, we do not really need to assume that $\mathbb{P}$ is ergodic, a stationary $\mathbb{P}$ will do, however our assumption is made out of habit. In fact even for the existence of the density of states one need not have the ergodicity assumption, if we follow the method of proof we use in Propositions 2.3, 2.5. We first define the density of states to be the average spectral measure with respect to $\delta_{0}$, show by invariance that it is the same as that for $\delta_{n}$. In the event this measure happens to be continuous, we show that the averages over space and randomness of the finite dimensional spectral measure converge to the density of states.

We start with some results leading to proving some symmetry properties of the measure $\rho$. 
Lemma 2.1. Suppose $(\Omega, \mathcal{B}, \mathbb{P})$ is a probability space and $M^{\omega}, N^{\omega}$ are finite dimensional matrices which are weakly measurable. Then the product $M^{\omega} N^{\omega}$ is weakly measurable.

Proof: We note that for matrices weak measurability is equivalent to measurability of each of the matrix entries as functions of $\omega$. Since the entries of product of matrices is a sum of products of entries of each of the matrices and since products and sums of measurable functions are measurable, the lemma follows.

Lemma 2.2. Suppose $P$ is an orthogonal projection on a separable Hilbert space $\mathcal{H}$ and $\left\{f_{n}\right\}$ is an orthonormal basis for $\mathcal{H}$. Then the following are equivalent.

1. $P=0$.

2. $\left\langle f_{n}, P f_{n}\right\rangle=0$ for all $n$.

Proof: We will show only (2) implies (1) since the other part follows by definition. Using the Cauchy-Schwarz inequality we see that for any $f, g \in \mathcal{H}$,

$$
\langle f, P f\rangle=0=\langle g, P g\rangle \Longrightarrow\langle f, P g\rangle=0=\langle g, P f\rangle \text {. }
$$

Since $f_{n}$ is an orthonormal basis for $\mathcal{H}$, finite linear span of the $f_{n} \mathrm{~s}$ is a total set, so the result follows if we show that

$$
\left\langle f_{n}, P f_{m}\right\rangle=0, \text { for all } m, n \text {. }
$$

For any pair of indices $m, n$, this follows from the previous inequality since $\left\langle f_{n}, P f_{n}\right\rangle=0=\left\langle f_{m}, P f_{m}\right\rangle$, by assumption.

Proposition 2.3. Consider the operators $H_{\omega}^{ \pm}$satisfying the Hypothesis 1.1. Then given any $a, b \in \mathbb{R}$, we have

$$
\mathbb{P}\left(\left\{\omega: E_{H_{\omega}^{ \pm}}(\{a, b\}) \neq 0\right\}\right)=0 .
$$

Proof: We consider the + case, the other one has a similar proof. Suppose the conclusion of the proposition is not valid so that, by Lemma 2.2, the set

$$
S=\left\{\omega:\left\langle\delta_{n}, E_{H_{\omega}^{+}}(\{a, b\}) \delta_{n}\right\rangle \neq 0 \text { for some } n \in \mathbb{Z}^{d}\right\}
$$


satisfies $\mathbb{P}(S) \neq 0$. Therefore for $\omega \in S$ we have,

$$
f(\omega)=\sum_{n \in \mathbb{Z}^{d}} 2^{-|n|}\left\langle\delta_{n}, E_{H_{\omega}^{+}}(\{a, b\}) \delta_{n}\right\rangle>0 .
$$

Let $K=\sum_{n \in \mathbb{Z}^{d}} 2^{-|n|}$, then we have using the invariance of the measure $\mathbb{P}$ under the action of $\mathbb{Z}^{d}$, in the second equality below,

$$
\begin{array}{r}
K n_{+}(\{a, b\})=\sum_{n \in \mathbb{Z}^{d}} 2^{-|n|} \mathbb{E}\left(\left\langle\delta_{0}, E_{H_{\omega}^{+}}(\{a, b\}) \delta_{0}\right\rangle\right) \\
=\sum_{n \in \mathbb{Z}^{d}} 2^{-|n|} \mathbb{E}\left(\left\langle\delta_{n}, E_{H_{\omega}^{+}}(\{a, b\}) \delta_{n}\right\rangle\right) \\
=\mathbb{E}\left(\sum_{n \in \mathbb{Z}^{d}} 2^{-|n|}\left\langle\delta_{n}, E_{H_{\omega}^{+}}(\{a, b\}) \delta_{n}\right\rangle\right) \\
=\mathbb{E}(f(\omega)) \geq \int_{S} f(\omega) d \mathbb{P}(\omega)>0 .
\end{array}
$$

This contradicts the continuity of the measure $n_{+}$, assured under Hypothesis 1.1(4), hence $S$ must have measure zero.

Lemma 2.4. Consider the operators $H_{\Lambda, \omega}^{ \pm}, H_{\omega}^{ \pm}$satisfying the Hypothesis 1.1. Let $K^{\omega}$ be a weakly measurable, uniformly bounded (in $\omega$ ) operators satisfying $U_{i}^{*} K^{\omega} U_{i}=K^{T_{i} \omega}$, where $U_{i}, T_{i}$ are as in Hypothesis 1.1(1). Then we have for arbitrary but fixed $A \in \mathcal{B}$ and for each finite $\Lambda \subset \mathbb{Z}^{d}$ :

1. $E_{H_{\Lambda, \omega}^{ \pm}}(A) K^{\omega}$ is trace class for all $\omega$.

2. We have :

$$
\operatorname{Tr}\left(E_{H_{\Lambda, \omega}^{ \pm}}(A) K^{\omega}\right)=\operatorname{Tr}\left(K^{\omega} E_{H_{\Lambda, \omega}^{ \pm}}(A)\right)=\operatorname{Tr}\left(E_{H_{\Lambda, \omega}^{ \pm}}(A) K^{\omega} E_{H_{\Lambda, \omega}^{ \pm}}(A)\right) .
$$

3. We also have :

$$
\frac{1}{|\Lambda|} \mathbb{E}\left(\operatorname{Tr}\left(E_{H_{\Lambda, \omega}^{ \pm}}(A) K^{\omega}\right)\right)=\mathbb{E}\left(\left\langle\delta_{0}, E_{H_{\Lambda, \omega}^{ \pm}}(A) K^{\omega} \delta_{0}\right\rangle\right)
$$

and

$$
\frac{1}{|\Lambda|} \mathbb{E}\left(\operatorname{Tr}\left(E_{H_{\Lambda, \omega}^{ \pm}}(A) K^{\omega} E_{H_{\Lambda, \omega}^{ \pm}}(A)\right)\right)=\mathbb{E}\left(\left\langle\delta_{0}, E_{H_{\Lambda, \omega}^{ \pm}}(A) K^{\omega} E_{H_{\Lambda, \omega}^{ \pm}}(A) \delta_{0}\right\rangle\right)
$$


Proof: (1) For any finite $\Lambda, H_{\Lambda, \omega}$ is a finite dimensional matrix and hence $E_{H_{\Lambda, \omega}^{ \pm}}(A)$ is a finite rank operator, so the product of $K^{\omega}$ with it is also trace class.

(2) These are simple properties of trace, namely, $\operatorname{Tr}(M N)=\operatorname{Tr}(N M)=$ $\operatorname{Tr}(M N M)$, for valid for bounded $N$ and trace class $M$ such that $M^{2}=M$.

(3) These relations are proved by writing the trace explicitly in terms of the basis vectors $\delta_{n}$, using $U_{i}^{*} K^{\omega} U_{i}=K^{T_{i} \omega}, U_{i}^{*} E_{H_{\Lambda, \omega}^{ \pm}}(A) U_{i}=E_{H_{\Lambda, T_{i} \omega}^{ \pm}}(A)$ and using the invariance of $\mathbb{P}$ under the action $T_{i}$.

We are now ready to state some properties of the measure $\rho$.

Proposition 2.5. Consider the operators $H_{\omega}^{ \pm}$, with $\omega \in \operatorname{supp}(\mathbb{P})$ and let $\rho$ be as in equation (5). Then for any borel subsets $B, C$ of $\mathbb{R}$,

1. $\rho(B \times C)=\mathbb{E}\left(\left\langle\delta_{0}, E_{H_{\omega}^{-}}(C) E_{H_{\omega}^{+}}(B) \delta_{0}\right\rangle\right)$

2. $\rho(B \times C)=\mathbb{E}\left(\left\langle\delta_{0}, E_{H_{\omega}^{-}}(C) E_{H_{\omega}^{+}}(B) E_{H_{\omega}^{-}}(C) \delta_{0}\right\rangle\right)$

3. $\rho(B \times C)=\mathbb{E}\left(\left\langle\delta_{0}, E_{H_{\omega}^{+}}(B) E_{H_{\omega}^{-}}(C) E_{H_{\omega}^{+}}(B) \delta_{0}\right\rangle\right)$

4. The following inequalities are valid

$$
\rho(B \times C) \leq n_{+}(B), \rho(B \times C) \leq n_{-}(C) .
$$

Proof: The inequalities in (4) follows from the the equalities (2), (3) and the fact that for any Borel set $B, E_{H_{\omega}^{ \pm}}(B)$ are orthogonal projections so $E_{H_{\omega}^{ \pm}}(B) \leq I, I$ being the identity operator.

(1) We consider $\mathcal{I}=\mathbb{R} \cup\{(a, b]: a, b \in \mathbb{R}\} \cup\{(a, \infty): a \in \mathbb{R}\} \cup\{(-\infty, a]:$ $a \in \mathbb{R}\}$. Let $B, C \in \mathcal{I}$ We have the inequality (by Cauchy-Schwarz)

$$
\begin{gathered}
\left|\mathbb{E}\left(\left\langle\delta_{0}, E_{H_{\Lambda, \omega}^{-}}(C) E_{H_{\Lambda, \omega}^{+}}(B) \delta_{0}\right\rangle\right)-\mathbb{E}\left(\left\langle\delta_{0}, E_{H_{\omega}^{-}}(C) E_{H_{\omega}^{+}}(B) \delta_{0}\right\rangle\right)\right| \\
\leq\left(\mathbb{E}\left(\left\|\left(E_{H_{\Lambda, \omega}^{-}}(C)-E_{H_{\omega}^{-}}(C)\right) \delta_{0}\right\|^{2}\right)\right)^{\frac{1}{2}}\left(\mathbb{E}\left(\left\|\left(E_{H_{\Lambda, \omega}^{-}}(B)-E_{H_{\omega}^{-}}(B)\right) \delta_{0}\right\|^{2}\right)\right)^{\frac{1}{2}}
\end{gathered}
$$

The operators $H_{\Lambda, \omega}^{ \pm}$converge in the strong resolvent sense for each $\omega \in \Omega$. Let $\partial B=\{a, b\}, \partial C=\{c, d\}$. By Proposition 2.3, we know that there is a set $\Omega(B, C)$ of full measure on which $E_{H_{\omega}^{+}}(\{a, b\})=0, E_{H_{\omega}^{-}}(\{c, d\})=0$. (If $a$ is not finite but $b$ is finite, then the statement would be $E_{H_{\omega}^{+}}(\{b\})=0$ and so on to cover all the elements of $\mathcal{I}$.) 
So $E_{H_{\Lambda, \omega}^{+}}(B)$ and $E_{H_{\Lambda, \omega}^{-}}(C)$ converge strongly point wise in $\omega \in \Omega(B, C)$, by Theorem VIII.24(2) of Reed-Simon [16].

Therefore using Lebesgue dominated convergence theorem we see that the right hand side of equation (10) converges to 0.

This shows that (1) is valid for the class of sets $B, C \in \mathcal{I}$. Since the measure $\rho$ is uniquely extended from this class of sets (see example 2.7.3, Parthasarathy [15] ), the relations now extend to all borel sets.

The proof of (2) and (3) are similar, so we show only (2) below.

(2) Again as in the previous argument, it is enough to consider $B, C \in \mathcal{I}$. We use the relation

$$
\begin{aligned}
& \left|\mathbb{E}\left(\left\langle\delta_{0}, E_{H_{\Lambda, \omega}^{-}}(C) E_{H_{\omega}^{+}}(B) E_{H_{\Lambda, \omega}^{-}}(C) \delta_{0}\right\rangle\right)-\mathbb{E}\left(\left\langle\delta_{0}, E_{H_{\omega}^{-}}(C) E_{H_{\omega}^{+}}(B) E_{H_{\omega}^{-}}(C) \delta_{0}\right\rangle\right)\right| \\
& \leq\left(\mathbb{E}\left(\left\|\left(E_{H_{\Lambda, \omega}^{-}}(C)-E_{H_{\omega}^{-}}(C)\right) \delta_{0}\right\|^{2}\right)\right)^{\frac{1}{2}}\left(\mathbb{E}\left(\left\|\left(E_{H_{\Lambda, \omega}^{-}}(C)-E_{H_{\omega}^{-}}(C)\right) \delta_{0}\right\|^{2}\right)\right)^{\frac{1}{2}}
\end{aligned}
$$

Therefore as before the right hand side above goes to zero as $|\Lambda| \rightarrow \infty$, via a choice of $\Omega(B, C)$ on which the integrands on the right hand side go to zero point wise and then the integral itself goes to zero using LDCT.

For each $\Lambda$ we have, by Lemma $2.4(2,3)$,

$$
\mathbb{E}\left(\left\langle\delta_{0}, E_{H_{\Lambda, \omega}^{-}}(C) E_{H_{\omega}^{+}}(B) E_{H_{\Lambda, \omega}^{-}}(C) \delta_{0}\right\rangle\right)=\mathbb{E}\left(\left\langle\delta_{0}, E_{H_{\omega}^{+}}(B) E_{H_{\Lambda, \omega}^{-}}(C) \delta_{0}\right\rangle\right) .
$$

The left and right hand sides of the above equation converge as $|\Lambda| \rightarrow \infty$ respectively to the left and the right hand sides of the equation

$$
\mathbb{E}\left(\left\langle\delta_{0}, E_{H_{\omega}^{-}}(C) E_{H_{\omega}^{+}}(B) E_{H_{\omega}^{-}}(C) \delta_{0}\right\rangle\right)=\rho(B \times C),
$$

arguing as before. Hence the result.

Lemma 2.6. Consider $H_{\omega}^{ \pm}$satisfying Hypothesis 1.1. Then, $n_{+}=n_{-}$and in this case $\rho$ is symmetric, i.e. $\rho(A \times B)=\rho(B \times A)$.

Proof: The hypothesis 1.1(2) says that for any integrable function $f, \mathbb{E}(f(\omega))=$ $\mathbb{E}(f(R \omega))$. Therefore taking $f(\omega)=\left\langle\delta_{0}, E_{H_{\omega}^{+}}(B) \delta_{0}\right\rangle$, for a fixed borel set $B$, we see that it is integrable and satisfies $f(R \omega)=\left\langle\delta_{0}, E_{H_{\omega}^{-}}(B) \delta_{0}\right\rangle$. Therefore $n_{+}(B)=\mathbb{E}(f(\omega))=\mathbb{E}(f(R \omega))=n_{-}(B)$. This being valid for any borel set $B$ the measures $n_{+}$and $n_{-}$agree. 
The symmetry of $\rho$ follows from the following equalities, using the invariance of $\mathbb{P}$ under $R$.

$$
\begin{aligned}
\rho(B \times C) & =\mathbb{E}\left(\left\langle\delta_{0}, E_{H_{\omega}^{+}}(B) E_{H_{\omega}^{-}}(C) \delta_{0}\right\rangle\right) \\
& =\mathbb{E}\left(\left\langle\delta_{0}, E_{H_{R \omega}^{-}}(B) E_{H_{R \omega}^{+}}(C) \delta_{0}\right\rangle\right) \\
& =\mathbb{E}\left(\left\langle\delta_{0}, E_{H_{\omega}^{-}}(B) E_{H_{\omega}^{+}}(C) \delta_{0}\right\rangle\right)=\rho(C \times B) .
\end{aligned}
$$

In the following we shall denote the marginals of $\rho \circ T^{-1}$ by,

$$
\nu_{1}=\frac{1}{2} \rho \circ T^{-1}(B \times \mathbb{R}), \nu_{2}=\frac{1}{2} \rho \circ T^{-1}(\mathbb{R} \times B) .
$$

Then, clearly

$$
A_{a s}(\lambda)=2 \nu_{1}((-\infty, \lambda]) \text { and } A_{s}(\lambda)=\left(\nu_{1}+\nu_{2}\right)((-\infty, \lambda]) .
$$

Remark 2.7. The measure $\rho$ is quite nice and we can say more about it. We shall denote by $B_{a}(x)$ a ball of radius a with centre $x \in \mathbb{R}^{2}$. We denote by $\nu$ the marginal $\rho(\cdot \times \mathbb{R})$ and note that $\nu=n_{+}$. In the case when $\rho(B \times \mathbb{R})=$ $\rho(\mathbb{R} \times B)$ for all borel $B$, then we have $\nu(B)=n_{+}(B)=n_{-}(B)$, from the definitions of $n_{ \pm}, \rho$ and $\nu$.

Definition 2.8. Given a probability measure $\mu$ and a bounded continuous function $h$ on $[r, \infty)$, positive on $(r, \infty)$ and vanishing at $r$, we say that $\mu$ has modulus of continuity $h$ at a point $x$ if

$$
\limsup _{a>0} \frac{\mu(x-a, x+a)}{h(a+r)}<\infty .
$$

We say that $\mu$ is uniformly $h$-continuous if the above condition is valid independent of $x$.

Examples 2.9. 1. Let $r=0, h(x)=x^{\alpha}, 0 \leq x \leq 1, h(x)=1, x>1$ for some $0<\alpha \leq 1$. Then $h$-continuity of $\mu$ for this $h$ is called $\alpha$-Hölder continuity.

2. If $r=1$ and $h(x)=(\ln (x))^{\alpha}, 1 \leq x \leq 2$ and some positive bounded continuous function on $(2, \infty)$ then $h$-continuity for this $h$ is called $\alpha$ log Hölder continuity. 
3. Let $r=0$. If $\nu$ is a probability measure and we take $h(a)=\nu((y-a, y+a)), y \in$ $\mathbb{R}$, then $h$-continuity with this $h$ means the modulus of continuity of $\mu$ at $x$ is the same as that of $\nu$ at $y$.

Remark 2.10. In the theorems below we will only present the case when $r=0$, the theorems easily follow even when we take $r \neq 0$ by taking $h(\cdot+r)$ to replace $h$.

Theorem 2.11. Consider $H_{\omega}^{ \pm}$satisfying Hypothesis 1.1. Suppose the density of states $n=n_{+}=n_{-}$is uniformly $h$-continuous for some $h$ as in Definition 2.8 with $r=0$. Then

$$
\rho\left(B_{a}(x)\right) \leq C h(a) .
$$

Proof: We consider the function $\psi(x)=\frac{1}{1+\|x\|^{2}}, x \in \mathbb{R}^{2}$, where $\|x\|^{2}=$ $x_{1}^{2}+x_{2}^{2}, x=\left(x_{1}, x_{2}\right)$. Then $\psi$ is integrable with respect to the probability measure $\rho$ on $\mathbb{R}^{2}$. This $\psi$ satisfies $\psi(x) \geq \frac{1}{2}$, whenever $\|x\| \leq 1$. So taking $\delta=1$ in Theorem 3.1, it is enough to show that

$$
\limsup _{a>0} \frac{1}{h(a)} \int \psi_{a}(y-x) d \rho(y)<\infty
$$

To see this we note that

$$
\psi_{a}(y-x) \leq \frac{1}{\left(1+\frac{\left(y_{1}-x_{1}\right)^{2}}{a^{2}}\right)},
$$

so that

$$
\begin{aligned}
\frac{1}{h(a)} \int \psi_{a}(y-x) d \rho(y) & \leq \frac{1}{h(a)} \int \frac{1}{\left(1+\frac{\left(y_{1}-x_{1}\right)^{2}}{a^{2}}\right)} d \nu\left(y_{1}\right) \\
& =\frac{1}{h(a)} \int \phi_{a}\left(y_{1}-x_{1}\right) d n\left(y_{1}\right)
\end{aligned}
$$

where we have integrated over the variable $y_{2}$ on the right hand side and used the definition of the measure $\nu$, remark 2.7 and have taken $\phi(y)=$ $1 /\left(1+y^{2}\right), \phi_{a}(y)=\phi(y / a), y \in \mathbb{R}$.

Then using Theorem 3.3, we see that the limsup of the right had side is finite for all $x_{1}$ once $n$ is uniformly $h$-continuous. Therefore the limsup of the left hand side is finite for all $x$.

This theorem shows that $\rho$ has no atoms. The marginals of $\rho$, namely $\rho(A \times \mathbb{R})$ and $\rho(\mathbb{R} \times A)$ both equal the density of states and hence are continuous if the density of states has no atoms. 
However it is possible that some marginal taken along other directions in $\mathbb{R}^{2}$ may have atoms (for example if $\rho$ is supported on a straight line and is absolutely continuous on that line, as a one dimensional measure, then the marginal taken orthogonal to this line would have an atom).

Theorem 2.12. Consider $H_{\omega}^{ \pm}$satisfying Hypothesis 1.1 and suppose the density of states $n$ is uniformly h-continuous for some $h$ as in Definition 2.8.

If $\nu_{1}$ or $\nu_{2}$ defined in equation (12) has an atom, then the function $A_{s}(\lambda)$ has a uniformly h-continuous component.

Proof: We first note that $\rho \circ T^{-1}(A \times B)$ is a finite positive measure as a function of $A$ for a fixed $B$ and as a function of $B$ for a fixed $A$, whenever $\rho \circ T^{-1}(A \times B) \neq 0$ for some pair $A, B$. We also note that if $\rho \circ T^{-1}(A \times$ $\mathbb{R}) \neq 0$, for a given $A$, then there must be a borel set $B \subsetneq \mathbb{R}$, such that $\rho \circ T^{-1}(A \times B) \neq 0$ (otherwise we can take one such $B$ and taking the union of $B$ and $B^{c}$, conclude that $\rho \circ T^{-1}(A \times \mathbb{R})=0$. A similar statement is valid when $\rho(\mathbb{R} \times B) \neq 0$.)

Therefore if the marginal $\rho \circ T^{-1}(A \times \mathbb{R})$ has an atom at a point $x$, then we can decompose the other marginal measure $\rho \circ T^{-1}(\mathbb{R} \times B)$ as

$$
\rho \circ T^{-1}(\mathbb{R} \times B)=\rho \circ T^{-1}(\mathbb{R} \backslash\{x\} \times B)+\rho \circ T^{-1}(\{x\} \times B) .
$$

Let $S_{\rho}$ denotes a finite subset of the set of atoms of $\rho \circ T^{-1}(A \times \mathbb{R})$, then we can write

$$
\begin{gathered}
\rho \circ T^{-1}(\mathbb{R} \times B)=\rho \circ T^{-1}\left(\mathbb{R} \backslash S_{\rho} \times B\right)+\rho \circ T^{-1}\left(S_{\rho} \times B\right) \\
=\rho_{1}(B)+\rho_{2}(B) .
\end{gathered}
$$

Similarly if $S_{\sigma}$ is some finite subset of the set of atoms of $\rho \circ T^{-1}(\mathbb{R} \times B)$, then we can write

$$
\begin{gathered}
\rho \circ T^{-1}(A \times \mathbb{R})=\rho \circ T^{-1}\left(A \times \mathbb{R} \backslash S_{\sigma}\right)+\rho \circ T^{-1}\left(A \times S_{\sigma}\right) \\
=\sigma_{1}(A)+\sigma_{2}(A) .
\end{gathered}
$$

We have for each $A, B$, the following relations, which is easy to see from the above argument.

$$
\begin{aligned}
& \rho_{2}(B)=\sum_{x \in S_{\rho}} \rho \circ T^{-1}(\{x\} \times B), \\
& \sigma_{2}(B)=\sum_{x \in S_{\sigma}} \rho \circ T^{-1}(A \times\{x\}) .
\end{aligned}
$$


Using the decomposition in equations $(15,16)$, we can write

$$
\begin{gathered}
A_{s}(\lambda)=\frac{1}{2}\left(\rho_{1}+\sigma_{1}\right) \\
((-\infty, \lambda])+\frac{1}{2}\left(\rho_{2}+\sigma_{2}\right)((-\infty, \lambda]) \\
=A_{s, 1}(\lambda)+A_{s, 2}(\lambda),
\end{gathered}
$$

where $A_{s, 1}, A_{s, 2}$ are non-zero functions, as seen by the preceding arguments.

Now the result follows from Lemma 2.13 below.

Lemma 2.13. Assume the conditions of theorem 2.12. Consider the function $A_{s, 2}$ defined in equation (17). If the density of states $n$ is uniformly $h$-continuous for some $h$ (as in Definition 2.8), then $A_{s, 2}$ is uniformly $h$ continuous for the same $h$.

Proof: We will prove that $\rho_{2}$ is uniformly $h$-continuous, the proof for $\sigma_{2}$ is similar. From these two statements the uniform $h$-continuity of $A_{s, 2}(\lambda)$ is clear. Let the cardinality of $S_{\rho}$ be $N$ and let $E_{1}, \ldots, E_{N}$ be the elements of $S_{\rho}$. Then

$$
\begin{aligned}
& \frac{1}{h(a)} \int \phi_{a}(y-x) d \rho_{2}(y)=\sum_{j=1}^{N} \frac{1}{h(a)} \int \phi_{a}(y-x) d \rho \circ T^{-1}\left(E_{j}, y\right) \\
& =\sum_{j=1}^{N} \frac{1}{h(a)} \int_{T\left(\left\{E_{j}\right\} \times \mathbb{R}\right)} \phi_{a}\left(w-\left(x+E_{j}\right)\right) d \rho\left(\frac{w}{\sqrt{2}}, \frac{w-2 E_{j}}{\sqrt{2}}\right)
\end{aligned}
$$

Since $T\left(\left\{E_{j}\right\} \times \mathbb{R}\right) \subset \mathbb{R} \times \mathbb{R}$, the right hand side is bounded by

$$
\begin{aligned}
& \sum_{j=1}^{N} \frac{1}{h(a)} \int_{\mathbb{R} \times \mathbb{R}} \phi_{a}\left(w-\left(x+E_{j}\right)\right) d \rho\left(\frac{w}{\sqrt{2}}, \frac{z}{\sqrt{2}}\right) \\
& =\sum_{j=1}^{N} \frac{1}{h(a)} \int_{\mathbb{R}} \phi_{a}\left(\sqrt{2} w-\left(x+E_{j}\right)\right) d n(w),
\end{aligned}
$$

where we used the fact that $d \rho(\cdot, \mathbb{R})=d n(\cdot)$. The uniform $h$-continuity of the density of states $n$ shows that the right hand side is bounded uniformly in $x$, proving the lemma.

Acknowledgement: I thank Prof Werner Kirsch for going through the paper and his comments.

\section{Appendix}

We present here some results that we use in the main part, whose proofs are essentially available elsewhere. 
We have an abstract theorem that extends the theorem of Jensen-Krishna in [6]. In the following let $(X,\|\cdot\|)$ be a normed vector space over complex numbers and $\rho$ a probability measure on $X$ with respect to the borel $\sigma$ algebra. Denote by $B_{a}(x)$ the ball with centre $x$ of radius $a$. Let $\psi$ be a positive bounded continuous function on $X$ taking value 1 at 0 . Denote by $\psi_{a}(x)=\psi(x / a), a>0$.

Theorem 3.1. Let $h$ be a function as in Definition 2.8. Suppose

$$
\limsup _{a>0} \frac{1}{h(a)} \int \psi_{a}(y-x) d \rho(y)<\infty
$$

Then, there are constants $C, \delta>0$, depending upon $\psi$, such that

$$
\rho\left(B_{a \delta}(x)\right) \leq C h(a), a>0, x \in X .
$$

Proof: Since $\psi$ is continuous and is 1 at 0 , there is a $\delta>0$ such that $\psi(y) \geq \frac{1}{2}$, whenever $\|x\|<\delta$. So we have

$$
\frac{1}{h(a)} \int \psi_{a}(y-x) d \rho(y) \geq \frac{1}{2 h(a)} \rho\left(B_{a \delta}(x)\right) .
$$

We have used the fact that $\|x\| / a \leq \delta \Longleftrightarrow\|x\| \leq a \delta$. Taking sup first on the left hand side, which is finite since the limsup of the left hand side is finite by assumption, and then taking sup over $a$, for a fixed $\delta$ on the right hand side shows that the right hand side is finite for all $x$.

Given a function $\psi$ satisfying:

Hypothesis 3.2. Let $\psi$ be a continuous function on $\mathbb{R}$ with $\psi(0)=1$ and $A_{\psi}=\int \psi(x) d x \neq 0$. Further assume that

1. $\psi$ is bounded and positive.

2. $\psi$ is differentiable, even and satisfies

$$
|\psi(x)|+\left|x \psi^{\prime}(x)\right| \leq\langle x\rangle^{-\delta} \text {, for some } \delta>1,
$$

where $\langle x\rangle=\left(1+x^{2}\right)^{1 / 2}$.

We set

$$
C_{\mu, \psi}^{h}=\limsup _{a>0} \frac{\psi_{a} * \mu}{h(a)}(x), D_{\mu, \psi}^{h}=\limsup _{a>0} \frac{\mu((x-a, x+a))}{h(a)}(x),
$$


Theorem 3.3. Let $\mu$ be a probability measure and let $\psi$ satisfy the Hypothesis 3.2. Then $C_{\mu, \psi}^{h}$ is finite for any $x$, iff $D_{\mu}^{h}(x)$ is finite for the same $x$.

Proof: The proof of this theorem proceeds on the lines of theorem 1.3.6 of [6] with the $a^{\alpha}$ there replaced by $h(a)$ and going through the arguments almost verbatim.

\section{References}

[1] M.S. Atoyan, E.M. Kazaryan, H.A. Sarkisyan: Interband light absorption in parabolic quantum dot in the presence of electrical and magnetic fields, Physica E: Low-dimensional Systems and Nanostructures Vol 31, 83-85 (2006).

[2] A. Avila and D. Damanik Absolute continuity of the integrated density of states for the almost Mathieu operator with non-critical coupling, Invent. Math., Vol 172, 439-453 (2008).

[3] R. Carmona, J. Lacroix: Spectral Theory of Random Schrödinger Operators, (Birkhäuser Verlag, Boston 1990)

[4] H. Cycone, R. Froese, W. Kirsch and B. Simon: Schrödinger Operators, Texts and Monographs in Physic (Springer Verlag, 1985)

[5] J.M. Combes, P. Hislop and F. Klopp: An optimal Wegner estimate and its application to the global continuity of the integrated density of states for random Schrödinger operators, Duke Math. J., Vol 140, 469-498 (2007).

[6] M. Demuth and M. Krishna: Determining spectra in Quantum Theory, Progress in Mathematical Physics Vol 44, Birkhauser, Boston, 2005

[7] A.L. Efros: Density of states and interband absorption of light in strongly doped semiconductors, Semiconductors, Vol 16, 1209- (1982).

[8] W. Kirsch and B. Metzger: The Integrated Density of States for Random Schr dinger Operators, Spectral theory and mathematical physics: a Festschrift in honor of Barry Simon's 60th birthday, Proc. Sympos. Pure Math., Vol 76 649-696 (2007). 
[9] A. Figotin, L. Pastur: Spectra of Random and Almost-Periodic Operators (Springer Verlag, Berlin 1992)

[10] W. Kirsch and L.A.Pastur: The large-time asymptotics of some Wiener integrals and the interband light absorption coefficient in the deep fluctuation spectrum, Comm. Math. Phys., Vol 132, 365-382 (1990).

[11] W. Kirsch and L.A.Pastur : The interband light absorption coefficient in the weak disorder regime: an asymptotically exactly solvable model, J. Phys. A, Vol 27, 2527-2543 (1994).

[12] W. Kirsch, L.A.Pastur and H. Stork : Asymptotics of the interband light absorption coefficient near the band edge for an alloy-type model, J. Statist. Phys, Vol 92, 1173-1191 (1998)

[13] B. Khoruzenko, W. Kirsch and L.A.Pastur: The Interband Light Absorption Coefficient in the weak disorder regime: An asymptotically exactly solvable model, J. Phys. A: Math. Gen. Vol 27, 2527-2543 (1994).

[14] M. Krishna: Continuity of integrated density of states - independent randomness, Proc. Ind. Acad. Sci., Vol 173, 401-410 (2007).

[15] K.R. Parthasarathy: Introduction to Probability and Measure, Texts and Readings in Mathematics, Vol 33, Hindustan Book Agency, New Delhi (2005).

[16] M. Reed and B. Simon: Methods of Modern Mathematical Physics, Functional Analysis, Academic Press (1972).

[17] Ivan Veselic: Existence and Regularity Properties of the Integrated Density of States of Random Schrödinger Operators, Lecture Notes in Mathematics No 1917, Springer Verlag (2008). 